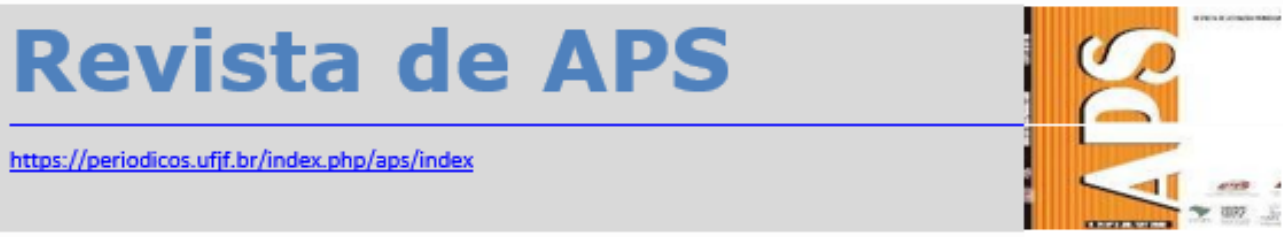

\title{
Dinâmica organizativa do acesso dos usuários aos serviços de saúde de média e alta complexidade
}

\author{
Organizational dynamics of user access to average and high complexity \\ health services
}

\author{
Mariana de Oliveira Araújo ${ }^{1}$, Maria Angela Alves do Nascimento², Bianca de Oliveira \\ Araujo $^{3}$
}

\section{RESUMO}

Estudo sobre o acesso dos usuários aos serviços de saúde de média e alta complexidade. Tem-se como objetivo analisar a dinâmica organizativa do acesso dos usuários aos serviços públicos de saúde de média e alta complexidade. Pesquisa qualitativa, crítica analítica, realizada com 26 participantes (profissionais, usuários e gestores dos serviços de saúde). As técnicas de coleta de dados foram: entrevista semiestruturada, observação sistemática e documentos. 0 método de análise foi o hermenêutico-dialético. O acesso aos serviços de saúde "transversalizam" as dimensões política, técnica e simbólica, as quais caminham para o atendimento das necessidades de saúde, apesar de ainda necessitar da participação da população para sua concretização como direito de cidadania.

PALAVRAS-CHAVE: Acesso aos serviços de saúde. Atenção à saúde. Sistema Único de Saúde.

\section{ABSTRACT}

Study about the user access to medium and high complexity health services. It has as objective to analyze the organizational dynamics of user access to medium and high complexity public health services. Qualitative research and critical analysis were conducted with 26 participants (professionals, users and managers of health services). The data collection techniques were semi-structured interview,

\footnotetext{
${ }^{1}$ Professora Assistente da Universidade Estadual de Feira de Santana (UEFS). Mestra e Doutoranda em Saúde Coletiva pela UEFS. E-mail: mariana-enf@hotmail.com.

2 Professora Emérita da Universidade Estadual de Feira de Santana (UEFS). Mestre em Enfermagem na área de Saúde Comunitária pela Universidade Federal do Rio de Janeiro (UFRJ), Doutora em Enfermagem pela Universidade de São Paulo (USP).

${ }^{3}$ Professora Assistente da Universidade Estadual de Feira de Santana (UEFS). Mestra e Doutoranda em Saúde Coletiva pela UEFS.
} 
systematic observation and document review. The method of analysis was hermeneutic-dialectic. The access to health services cross the political, technical and symbolic dimensions, which move to meet the health needs, although it still requires the participation of the population for its implementation as a citizenship right.

KEYWORDS: Health services accessibility. Health care. Unified Health System.

\section{INTRODUÇÃO}

O acesso aos serviços de saúde de maneira universal, equânime e integral se constitui como um direito de cidadania, garantido legalmente na Constituição Federal Brasileira de $1988,{ }^{1}$ a qual regulamenta e institui o Sistema Único de Saúde (SUS).

A partir de então, como preconiza o artigo 196 da Constituição Federal, em sua Seção II Da Saúde, a saúde passa a se constituir em "um direito de todos os cidadãos e dever do Estado", o qual deve ser garantido mediante políticas sociais e econômicas que objetivem reduzir o risco de doença e de outros agravos, bem como "promover o acesso universal e igualitário às ações e serviços para sua promoção, proteção e recuperação" ${ }^{1}$

Contudo, percebemos que apesar de o SUS garantir legalmente o acesso universal aos serviços de saúde, tal princípio não tem sido traduzido, efetivamente, na prática, em especial nas ações de média e alta complexidade. Por outro lado, a organização da rede dos serviços de saúde no Brasil tem vivido grandes mudanças com a introdução de programas novos e estratégicos, como o Programa Saúde da Família (PSF), atualmente nominado de Estratégia Saúde da Família (ESF), que mesmo com alguns avanços conquistados não tem consolidado a efetivação do acesso aos serviços disponibilizados pelo SUS, que ainda são desiguais e excludentes em algumas situações. ${ }^{2}$

Alguns estudos $2,4,5,6$ coadunam com essa discussão ao demonstrar que o acesso universal aos serviços de saúde não tem se efetivado na prática, caracterizados por uma assistência muitas vezes seletiva e excludente, diante de entraves para o ingresso do usuário ao sistema e a garantia de resolubilidade dos problemas de saúde apresentados pelos indivíduos e a coletividade.

Para tanto, neste estudo apresentamos como questão norteadora a seguinte premissa: como se processa a dinâmica organizativa do acesso (na perspectiva política, 
técnica e simbólica) dos usuários aos serviços de saúde de média e alta complexidade da rede SUS?

Por conseguinte, o estudo objetiva analisar a dinâmica organizativa do acesso dos usuários aos serviços públicos de saúde de média e alta complexidade.

No sentido de uma melhor compreensão sobre os serviços de média e alta complexidade definimos como média complexidade as ações que demandam a disponibilidade de profissionais especializados e o uso de recursos tecnológicos de apoio diagnóstico e terapêutico. Como alta complexidade, define-se um conjunto de procedimentos de alta tecnologia e alto custo com o objetivo de propiciar à população acesso aos serviços, integrando-os aos outros níveis de atenção à saúde. ${ }^{7}$

Para a análise da dinâmica organizativa do acesso dos usuários aos serviços de média e alta complexidade, definimos como bases orientadoras as dimensões de análise de acesso política, técnica e simbólica. ${ }^{2,8}$

A "dimensão política" é relativa ao desenvolvimento da consciência sanitária e da organização popular, trata-se da garantia do acesso como um direito do cidadão. A "dimensão técnica" diz respeito à utilização e às dificuldades encontradas pelo usuário no seu atendimento, bem como ao planejamento e à organização da rede de serviços, estando relacionada à integralidade da atenção, de modo a resolver o problema de saúde. A "dimensão simbólica" relaciona-se às representações sociais acerca da maneira como se organiza e como são disponibilizados os serviços de atenção à saúde..$^{2,8}$

Consideramos importante compreender a dinâmica organizativa do acesso dos usuários aos serviços públicos de saúde de média e alta complexidade, em suas dimensões técnica, política e simbólica, no intuito de contribuir com sua configuração nesses níveis de atenção e com a promoção da saúde como um direito de cidadania, conforme preconiza a Constituição Federal.

\section{MÉTODOS}

Trata-se de um estudo qualitativo, crítico-analítico, em uma perspectiva aproximada do método dialético, tendo como cenário Unidades de Saúde da Família (USF), policlínicas e hospitais públicos de um município de grande porte do interior da Bahia (Brasil). 
O critério de escolha de tal cidade se deve em razão do seu lugar de destaque na atenção à saúde de média e alta complexidade, visto que, de acordo com o Plano Diretor de Regionalização, como município sede da Macrorregião Centro-Leste, constitui-se referência para tais serviços. ${ }^{9}$

Os participantes deste estudo dividem-se em três grupos: Grupo I-profissionais de saúde (nove entrevistados), Grupo II - usuários (oito entrevistados) e Grupo III gestores/coordenadores dos serviços de saúde (nove entrevistados), totalizando 26 participantes. Utilizamos como técnicas de coleta de dados a entrevista semiestruturada, a observação sistemática e a análise de documentos. Na construção da análise dos dados os participantes são nominados nos fragmentos das suas falas a partir da ordem de cada entrevista realizada com a respectiva inicial da letra do grupo ao qual pertence. Desse modo, identificamos, por exemplo: Ent. 2P (profissional de saúde), Ent. 3U (usuário), Ent. $8 \mathrm{G}$ (gestor/ coordenador dos serviços de saúde).

O método de análise de dados utilizado foi o hermenêutico-dialético, por entendermos que possibilita o encontro de duas perspectivas teóricas que ultrapassam a descrição e fortalecem a subjetividade dos participantes do estudo. A análise de dados foi didaticamente dividida nos seguintes momentos: ordenação, classificação e análise final. A partir do confronto dos dados emergiram as três categorias discutidas a seguir.

Salientamos que a pesquisa foi iniciada após aprovação no Comitê de Ética em Pesquisa da Universidade Estadual de Feira de Santana (UEFS), no dia 23 de janeiro de 2013, segundo parecer no 188.191/2013 (CAAE 11789312.8.0000.0053).

\section{RESULTADOS}

A transversalidade das dimensões política, técnica e simbólica na organização do acesso

Os diversos sentidos e significados que caracterizam a palavra acesso "transversalizam" por aspectos que influenciam na sua concretização. O acesso aos serviços de saúde se insere em um tema multifacetado que envolve aspectos políticos, econômicos, sociais, organizativos, técnicos e simbólicos para o estabelecimento da universalização da atenção. ${ }^{10}$ 
A partir das entrevistas, das observações e dos documentos analisados percebemos que os sentidos/significados de acesso aos serviços de saúde apresentados pelos participantes deste estudo "transversalizam" a dimensão política, técnica e simbólica.

A "dimensão política" perpassa pela garantia do acesso como um direito de cidadania, a partir da viabilização de programas especiais, da pactuação entre as instâncias (estadual e municipal) e da participação e organização social.

Destacamos os programas especiais como artifícios da organização da assistência em saúde que em alguns momentos têm colaborado para a concretização do acesso aos serviços de média e alta complexidade, conforme apresentado na fala de alguns profissionais de saúde e gestores pesquisados.

Ademais, alguns documentos analisados ${ }^{11,12}$ apresentam no item Implantação e Implementação de Centros de Referência atividades que nos levam a pensar que os gestores têm buscado garantir um atendimento prioritário a grupos específicos que poderão facilitar o acesso aos serviços de média complexidade.

A ampliação do acesso aos serviços de média complexidade, como consultas e procedimentos especializados, bem como a articulação dos pontos da rede são aspectos desse nível de atenção, considerados imprescindíveis para a garantia da resolubilidade e integralidade da assistência. ${ }^{13}$

A participação e a organização social poderão colaborar ou não para formulação e implementação de políticas que atendam as demandas apresentadas por usuários, trabalhadores e gestores, como disposto na Lei $n$ o 8.142/1990. ${ }^{14}$

Segundo Rolim, Cruz e Sampaio,,15:140 o controle/participação social é considerado "uma das formas mais avançadas de democracia, pois determina uma nova relação entre o Estado e a sociedade, de maneira que as decisões sobre as ações na saúde deverão ser negociadas", essa característica se destaca entre os princípios do SUS em razão de sua relevância social e política, pois garante a participação da população no processo de formulação e controle das políticas públicas de saúde.

Entretanto, apesar da existência dos meios de concretização da participação social, as falas dos usuários demonstraram que eles desconhecem a existência de conselho local de saúde em sua comunidade e não participam dele. 
A "dimensão técnica" do acesso é relativa às ações desenvolvidas que buscam transformar o processo de trabalho em saúde a partir da "apreensão de dispositivos da integralidade da atenção: acolhimento, vínculo e autonomia da equipe e usuário, indicando um projeto terapêutico compartilhado". ${ }^{10: 2872}$

Acreditamos que a "dimensão técnica" do acesso aos serviços de saúde perpassa pela garantia da integralidade da atenção, promovida a partir do planejamento e da programação das ações, da organização da rede de serviços e do desenvolvimento de acolhimento, vínculo e compromisso.

Desse modo, um dos entrevistados considera que "[...] ter acesso aos serviços de saúde é você conseguir resolver seu problema na unidade que você procura [...] é [...] ter a resolutividade daquele problema [...]" (Ent. 12P), de modo a garantir a integralidade da atenção.

Para que os serviços estejam organizados e atendam às necessidades concretas da população o planejamento e a programação em saúde surgem como ferramentas importantes desse processo.

De modo divergente, as falas dos profissionais de saúde e gestores expressam que ora há um planejamento que possibilite uma maior integração entre os serviços ora ocorrem a partir de uma cobrança da gestão municipal. Para tanto, revelam que as necessidades da comunidade têm sido identificadas nas reuniões comunitárias, conforme modelo proposto para a construção do Plano Municipal de Saúde 2010-2013 ${ }^{11}$ e também da Programação Anual de Saúde. ${ }^{12}$

A "dimensão simbólica" do acesso adentra justamente no campo da subjetividade em sua dinâmica relacional, permeando a compreensão da cultura, da crença e dos valores de sujeitos e grupos sociais que vivem nos territórios onde os serviços de saúde estão organizados. ${ }^{10}$

Os valores, as crenças e a cultura de cada indivíduo influenciam a maneira como percebem e como qualificam determinados serviços. Por isso, para um dos usuários entrevistados o acesso perpassa pelo "[...] bom atendimento, porque [...] pela situação em que a gente se encontra, a gente quer ser melhor atendido" (Ent. 24U).

A partir das divergências, das convergências, das complementariedades e das diferenças encontradas nas falas dos grupos pesquisados, percebemos que essas dimensões "congregam para o atendimento das necessidades de saúde, tendo como 
imagem objetivo uma atenção responsável, integral, resolutiva, equânime e de qualidade". ${ }^{10: 2873}$

Práticas dos trabalhadores nos serviços de saúde de média e alta complexidade: um entrelaçamento entre as tecnologias de saúde

Na operacionalização do cuidado, objeto do trabalho em saúde, são necessários instrumentos ou tecnologias que podem ser utilizadas pelos profissionais de saúde, as quais podem ser classificadas como leves (tecnologias de relação, capazes de promover acolhimento, vínculo, compromisso e autonomia ao usuário), tecnologias leve-duras (saberes estruturados, como a clínica, a epidemiologia, entre outros) e tecnologias duras (equipamentos, máquinas, normas, estruturas organizacionais). ${ }^{16}$

A garantia da integralidade da atenção, o planejamento e a programação das ações de saúde; a organização da rede de serviço e o desenvolvimento do acolhimento, do vínculo e do compromisso são atividades técnicas ("dimensão técnica") realizadas por profissionais e gestores do SUS para a operacionalização do acesso aos serviços de saúde.

A prática dos profissionais de saúde para a operacionalização do acesso aos serviços de média e alta complexidade no município pesquisado em alguns momentos tem se voltado para o compromisso nas ações desenvolvidas, como verificado em algumas falas que expressam o empenho empreendido pelos profissionais para possibilitar que o acesso aos serviços de média e alta complexidade seja efetivado. Contudo, alguns usuários apresentam falas divergentes ao afirmarem que as ações de alguns profissionais não têm sido acolhedoras, o que se constitui em uma dificuldade para a promoção do acesso de média e alta complexidade.

Entretanto, durante nossa coleta de dados percebemos que o compromisso dos trabalhadores de saúde pôde ser observado tanto na Atenção Básica quanto em serviços de média e alta complexidade. Esse compromisso foi evidenciado na tentativa de marcar consultas e/ou cirurgias; no contato telefônico para comunicar as datas de agendamentos de consultas e/ou procedimentos vindos da Central de Regulação que já estavam próximas, entre outros.

Como destacado na fala de alguns usuários (Ent. 4U, 9U, $11 \mathrm{U}$ e 24U), a condução desse usuário pelos níveis de assistência à saúde na rede SUS tem sido facilitada pela existência de relatório de encaminhamento, guias e fichas de referência para as 
unidades as quais se destinam, pois permitem que o quadro clínico apresentado pelo indivíduo e os cuidados realizados na unidade de origem sejam de conhecimento do profissional de saúde que irá recebê-lo.

O fato de o usuário ser encaminhado pelo serviço de saúde a outro serviço acompanhado de uma guia ou um relatório se constitui uma facilidade para ingressar nos serviços de média e alta complexidade, funcionando como um "passaporte" ao sistema de referência e contrarreferência, como pudemos perceber durante a observação nos estabelecimentos pesquisados.

Por isso, acreditamos que mecanismos facilitadores do processo de referência e contrarreferência são fundamentais para a concretização da integralidade. Além disso, a humanização, a manutenção do vínculo entre paciente, família e sistema de saúde, a redução do tempo de internação e da exposição às complicações daí decorrentes, assim como a disponibilização de leitos e diminuição dos custos são consideradas consequências benéficas desse processo. ${ }^{17}$

\section{A relação público versus privado no acesso aos serviços de média e alta complexidade}

O acesso aos serviços de saúde é um caminho que o usuário deverá percorrer, desde a apresentação de uma necessidade ou de um problema de saúde específico até o ingresso no sistema.

Na organização dos serviços de saúde o acesso poderá se concretizar a partir de uma porta de entrada que teoricamente seria a Atenção Básica, e caso não encontre a resposta desejada ao seu problema nesse nível de atenção, o usuário "caminharia" pela média e pela alta complexidade buscando resolvê-lo. Entretanto, alguns estudos apontam que, na prática, outros serviços têm se instituído em porta de entrada do SUS. ${ }^{18,19}$

A porta de entrada do sistema tem se materializado a partir do problema que o usuário apresenta, escolhendo por onde deseja acessar os serviços. Na verdade, são diversas as entradas que pudemos identificar em observações e entrevistas (PSF, policlínica, Serviço de Atendimento Móvel de Urgência [SAMU], hospital) que permitem o usuário caminhar na rede de serviços em busca do atendimento de média e alta complexidade para dar resolubilidade às suas necessidades de saúde. Ainda 
acrescentamos como meio de ingresso nos níveis de maior complexidade a atenção particular, conforme a fala de alguns usuários (11U, $22 \mathrm{U}$ e $24 \mathrm{U})$.

A procura dos usuários pelos serviços particulares poderá estar associada à falta de resolubilidade dos serviços públicos frente às necessidades que apresenta naquele momento. No entanto, os Ent. $11 \mathrm{U}, 22 \mathrm{U}$ e $24 \mathrm{U}$ divergem dos Ent. 3U, $9 \mathrm{U}$ e $22 \mathrm{U}$, ao referirem à falta de resolubilidade e os entraves que têm encontrado para acessar os serviços de saúde de média e alta complexidade a partir dos serviços públicos, tendo que, muitas vezes, mesmo sem condições financeiras, procurar serviços de saúde privados.

Isso nos leva a reforçar que essa falta de resolubilidade e a demora em conseguir assistência no sistema público podem estar colaborando para que os usuários busquem por atendimento na rede privada. Outra justificativa para a procura do setor particular pode estar relacionada à demanda maior que à oferta de serviços no SUS, observada na prática.

As demandas expressam determinadas "carências" ou "necessidades" geradas pelo modo de produção e organização da sociedade. ${ }^{19}$

A demanda maior que a oferta de serviços e procedimentos de média e alta complexidade foi uma dificuldade/limite para a promoção do acesso referido pelos três grupos entrevistados [16G, 17G, 18G, 1U, 4U, 9U, 2P, 6P, 7P].

De acordo com os problemas priorizados pela população e pelo Núcleo de Apoio à Saúde da Família da Unidade Básica de Saúde (NASF/UBS), no Plano Municipal de Saúde $2010-2013^{11}$ o déficit de marcação de exames e consultas pela Central de Regulação foi elencado por várias unidades, interferindo diretamente na dinâmica de atendimento. No entanto, com vistas a solucionar esse problema, a Programação Anual de Saúde ${ }^{12}$ e o Plano Municipal de Saúde ${ }^{11}$ reforçam as ações propostas, como a ampliação da oferta de exames e/ou procedimentos de média e alta complexidade, bem como o aumento no quantitativo das cotas de marcação de consultas ou procedimentos pactuados com o estado.

Durante o período de coleta de dados observamos que o fluxo de atendimento da maioria das Unidades de Saúde da Família (USF), policlínicas e hospitais estudados é intenso e que os usuários têm encontrado dificuldades na marcação de procedimentos 
e/ou consultas especializadas nas UBS pela Central de Regulação, o que expressa que realmente a demanda pelos serviços tem sido maior que a oferta.

Acreditamos que a "identificação de barreiras econômicas, sociais, culturais e físicas" poderá colaborar para a garantia do acesso e "proporcionar equidade na dinâmica da organização dos serviços em seus diferentes níveis de complexidade do sistema". 10:2872

\section{DISCUSSÃO}

A lógica de organização do SUS pressupõe a constituição de uma rede de serviços hierarquizada e regionalizada de modo a permitir o acesso universal às ações e aos serviços de saúde, através do nível primário de atenção e/ou serviços especializados ambulatoriais e hospitalares, que busquem solucionar a necessidade de saúde apresentada pelo usuário.

Entretanto, discussões do acesso elencadas no estudo de Assis, Villa e Nascimento $^{2}$ destacam que, no Brasil, ainda se convive com o acesso seletivo, excludente e focalizado nos diferentes serviços públicos e privados.

Quando nos reportamos aos serviços de média e alta complexidade, vislumbramos de maneira mais evidente o quanto o acesso a esses serviços tem se conformado de maneira seletiva, excludente e focalizada, pois tem sido condicionado pelo poder de compra dos usuários, pela racionalização dos gastos e pela limitação do atendimento público a serviços e programas específicos.

Daí a importância de compreendermos como o acesso dos usuários aos serviços públicos de saúde de média e alta complexidade tem se organizado em suas dimensões técnica, política e simbólica, com o intuito de contribuir com sua configuração nesses níveis de atenção e a promoção da saúde como direito de cidadania, conforme preconiza a Constituição Federal.

Retomando alguns estudos, ${ }^{3,4,5,6}$ percebemos que a efetivação do acesso à saúde apresenta entraves e limites que precisam ser superados. Um desses limites seria a oferta/demanda, sendo necessária uma ampliação dos serviços ofertados que atendam às demandas da população usuária para proporcionar o desenvolvimento de ações de qualidade e resolutivas tanto na Atenção Básica, quanto de média e alta complexidade, 
a partir de práticas que ultrapassem o modelo de atenção tradicional - medicalizante, biologizante e profissionalmente centrado - para o modelo focado no usuário na qualidade de centro da atenção desenvolvida.

Diante da realidade do modelo médico hegemônico neoliberal que ainda prevalece nos serviços de saúde, e que se caracteriza por ser centrado na figura do médico, na doença e na "queixa-conduta", acreditamos que o acesso, ao ser concretizado como um direito, poderá contribuir com a transformação das práticas, tornando-as integrais, humanas e resolutivas, de modo a permitir e facilitar a entrada do usuário no SUS e a consequente resolubilidade do problema de saúde.

\section{CONCLUSÃO}

A dinâmica organizativa do acesso dos usuários aos serviços públicos de saúde de média e alta complexidade tem sido "transversalizada" pelos diversos sentidos e significados que o termo acesso representa para os profissionais de saúde, gestores e usuários do sistema, materializando-o nas dimensões política, técnica e simbólica.

Dialeticamente percebemos que as tecnologias leve, leve-dura e dura têm entremeado o processo de trabalho dos profissionais de saúde tanto da Atenção Básica, quanto de média e alta complexidade, para a promoção do acesso dos usuários aos serviços de saúde.

Destacamos como dificuldades e limites que os usuários têm encontrado para acessar os serviços de saúde de média e alta complexidade no município estudado e no cenário nacional, como demonstra outros estudos, a demanda por esses serviços ser maior que a oferta.

Deparamos com diversas portas de entradas utilizadas pelos usuários para acessar os serviços de média e alta complexidade. Além disso, a relação entre o público e o privado é constitutiva da organização dessa realidade. O acesso do usuário aos serviços e às práticas de média e alta complexidade ainda necessita, para a sua concretização, de um comprometimento de usuários, profissionais e gestores da saúde de modo a operacionalizá-lo como um direito de cidadania. 


\section{REFERÊNCIAS}

1. Brasil. Constituição da República Federativa do Brasil, 1988. Título VIII, Seção II, Artigos 196 a 200. Da Saúde. Brasília, DF: Senado Federal; 1988.

2. Assis MMA, Villa TCS, Nascimento, MAA. Acesso aos serviços de saúde: uma possibilidade a ser construída na prática. Ciên Saúde Colet 2003; 8(3): 815-823.

3. Corrêa ACP, Ferreira F, Cruz GSP, Pedrosa ICF. Acesso a serviços de saúde: olhar de usuários de uma unidade de saúde da família. Rev Gaúcha Enferm., Porto Alegre (RS) 2011 set; 32(3): 451-7.

4. Gomide MFS, Pinto IC, Figueiredo LA. Acessibilidade e demanda em uma Unidade de Pronto Atendimento: perspectiva do usuário. Acta Paul. Enferm, 2012; 25(spe2): 1925.

5. Puccini PT, Cornetta VK, Sahyom TZ, Fuentes ICP, Botta LMG, Puccini RF. Concepção de profissionais de saúde sobre o papel das unidades básicas nas redes de atenção do SUS/Brasil. Ciência \& Saúde Coletiva 2012; 17(11), 2941-2952.

6. Silva BFS, Benito GAV. A voz de gestores municipais sobre o acesso à saúde nas práticas de gestão. Ciência \& Saúde Coletiva, Rio de Janeiro 2013; 18(8), 21892200.

7. Brasil. O SUS de A a Z: garantindo saúde nos municípios. 3. Ed. Brasília: Ministério da Saúde; 2009.

8. Giovanella L, Fleury S. Universalidade da Atenção à Saúde: acesso como categoria de análise. In: Eibenschutz C, organizadora. Política de Saúde: o público e o privado. Rio de Janeiro: Fiocruz; 1995. p. 177-198.

9. Bahia. Plano Diretor de Regionalização. Secretaria de Saúde do Estado da Bahia. Regiões de Assistência em Saúde. [acesso em 2012 dez. 04]. Disponível em: http://www1.saude.ba.gov.br/mapa_bahia/.

10. Assis MMA, Abreu-de-Jesus WL. Acesso aos serviços de saúde: abordagens, conceitos, políticas e modelo de análise. Ciên Saúde Colet 2012; 17(11): 2865-2875.

11. Feira de Santana. Secretaria Municipal de Saúde. Plano Municipal de Saúde, exercício 2010-2013. Feira de Santana; 2010.

12. Feira de Santana. Secretaria Municipal de Saúde. Programação Anual de Saúde 2012. Feira de Santana; 2012.

13. Erdmann AL, Andrade SR, Mello ALSF, Drago LC. Secondary Health Care: best practices in network services. Rev. Latino-Am. Enfermagem [Internet]. jan.-fev. 2013 [acesso em: 2015 abr. 10];21(Spec):[08 telas]. Disponível em: http://www.scielo.br/pdf/rlae/v21nspe/pt_17.pdf.

14. Brasil. Ministério da Saúde. Legislação Federal, 1990. Lei N 8.142,1990. [acesso em 2012 dez. 04]. Disponível em: http://portal.saude.gov.br/portal/arquivos/pdf/Lei8142.pdf. 
15. Rolim LB, Cruz RSBLC, Sampaio KJAJ. Participação popular e o controle social como diretriz do SUS: uma revisão narrativa. Saúde em Debate [online] 2013; 37(96): 139-147.

16. Merhy EE. Saúde: cartografia do trabalho vivo. 2. ed. São Paulo: Hucitec; 2005.

17. Fratini JRG, Saupe R, Massaroli A. Referência e contrarreferência: contribuição para a integralidade em saúde. Ciên, Cuidado e Saúde 2008; 7(1): 065-072.

18. Oliveira LH, Mattos RA, Souza AIS. Cidadãos peregrinos: os "usuários" do SUS e os significados de sua demanda a prontos-socorros e hospitais no contexto de um processo de reorientação do modelo assistencial. Ciên Saúde Colet 2009; 14(5): 1929-1938.

19. Dubeux LS, Freese E, Felisberto E. Acesso a hospitais regionais de urgência 345 e emergência: abordagem aos usuários para avaliação do itinerário e dos obstáculos aos serviços de saúde. Physis: Revista de Saúde Coletiva 2013; 23(2): 345-368.

20. Silva JPV, Pinheiro R, Machado FRS. Necessidades, demanda e oferta: algumas contribuições sobre os sentidos, significados e valores na construção da integralidade na reforma do setor saúde. Saúde em Debate 2003; 27(65): 234-242.

Submissão: fevereiro de 2016.

Aprovação: outubro de 2018. 